\title{
Induction of Cross-resistance to ABCB1 Substrates in Venetoclax-resistant Human Leukemia HL60 Cells
}

\author{
YUKO NAKAYAMA ${ }^{1,2}$, KOHJI TAKARA $^{2,3}$, TETSUYA MINEGAKI ${ }^{4}$, \\ KAZUHIRO YAMAMOTO ${ }^{1,5}$, TOMOHIRO OMURA ${ }^{1,5}$ and IKUKO YANO ${ }^{1,5}$ \\ ${ }^{1}$ Department of Pharmaceutics, Kobe University Graduate School of Medicine, Kobe, Japan; \\ ${ }^{2}$ Department of Clinical Pharmaceutics, Faculty of Pharmaceutical Sciences, \\ Himeji Dokkyo University, Himeji, Japan; \\ ${ }^{3}$ Department of Pharmaceutics and Pharmaceutical Technology, \\ Faculty of Pharmaceutical Sciences, Hyogo University of Health Sciences, Kobe, Japan; \\ ${ }^{4}$ Department of Clinical Pharmacy, Kyoto Pharmaceutical University, Kyoto, Japan; \\ ${ }^{5}$ Department of Pharmacy, Kobe University Hospital, Kobe, Japan
}

\begin{abstract}
Background/Aim: Resistance to venetoclax, a selective inhibitor of BCL2 apoptosis regulator (BCL2), is regarded as a clinical problem. However, it is unclear whether resistance to venetoclax induces cross-resistance to other drugs. Materials and Methods: Venetoclax-resistant HL60/VEN cells were newly established through continuous exposure of human acute promyelocytic leukemia HL60 cells to venetoclax, and drug sensitivity, apoptotic activity, and $m R N A$ expression were compared between HL60 and HL60/VEN cells. Results: HL60/VEN cells displayed approximately 3-fold resistance to venetoclax, maintained their ability to synthesize DNA and had low apoptotic activity. HL60/VEN cells also exhibited diverse sensitivity to cytotoxic drugs, especially resistance to ATP binding cassette subfamily $B$ member 1 ( $A B C B 1)$ substrates, and up-regulation of $A B C B 1 \mathrm{mRNA}$. However, the sensitivity of HL60/VEN cells to venetoclax was not restored by ABCBI inhibitor. ABCB1-overexpressing cells did not show resistance to venetoclax. Conclusion: HL60/VEN cells exhibited upregulation of $A B C B 1$ in addition to an alteration in apoptotic activity, and cross-resistance to $A B C B 1$ substrates was clarified. However, sensitivity to venetoclax was hardly affected by $A B C B 1$.
\end{abstract}

This article is freely accessible online.

Correspondence to: Kohji Takara, Ph.D., Department of Pharmaceutics and Pharmaceutical Technology, Faculty of Pharmaceutical Sciences, Hyogo University of Health Sciences, 13-6 Minatojima, Chuo-ku, Kobe, 650-8530, Japan. Tel: +81 783043170, Fax: +81 783042870, e-mail: ko-takara@huhs.ac.jp

Key Words: Venetoclax, BCL2, resistance, ABC transporter, HL60 cells.
Venetoclax is a first-in-class, oral, selective inhibitor of BCL2 apoptosis regulator (BCL2) that is approved worldwide $(1,2)$. Venetoclax as monotherapy or in combination with rituximab is an effective treatment, provides durable responses in adults with recurrent/refractory chronic lymphocytic leukemia, and has a manageable safety profile in pivotal clinical trials (3-5). Trials are currently underway to expand its use for various other types of cancer, including multiple myeloma and chronic myeloid leukemia $(6,7)$.

BCL2 an anti-apoptotic protein is a regulator of cell death (i.e., apoptosis) (8). Evasion of apoptosis is one of the typical characteristics of cancer cells, and a change in expression of BCL2 family proteins has been reported in many cancer types (9-11). Therefore, current interest is focused on targeting BCL2 to treat various hematological malignancies, which are typically mediated by the intrinsic regulation of apoptosis or dysregulation of the mitochondrial pathway (12-14). Venetoclax binds and neutralizes BCL2 (BH3 domain), resulting in the release of cytochrome $c$ from the mitochondria and subsequent activation of the intrinsic apoptotic pathway through a caspase cleavage cascade $(8,15,16)$.

Multidrug resistance remains a significant problem in cancer chemotherapy $(17,18)$. Tumor cells have been found to acquire resistance not only to cytotoxic anticancer drugs, but also to molecular targeting agents. To date, the mechanisms of resistance to venetoclax have been investigated, and changes in molecular profiles have been found to occur in venetoclax-resistant cells (19). Although resistance to venetoclax is regarded as a clinical problem $(20,21)$, it is unclear whether resistance to venetoclax induces resistance to drugs besides venetoclax i.e., leads to cross-resistance. 
Here, a human acute promyelocytic leukemia cell line, HL60, as a leukemia cell model, was continuously exposed to venetoclax at a clinically achievable concentration, and a novel venetoclax-resistant cell (named as HL60/VEN) was established. The sensitivity to venetoclax and other anticancer drugs and the molecular changes in HL60/VEN cells were examined and compared with those in HL60 cells.

\section{Materials and Methods}

Chemicals. Venetoclax was purchased from Selleck Chemicals (Houston, TX, USA). Carboplatin, cisplatin, doxorubicin hydrochloride, etoposide, 5-fluorouracil, paclitaxel, vinblastine sulfate and vincristine sulfate were purchased from FUJIFILM Wako Pure Chemical Corp. (Osaka, Japan). 7-Ethyl-10-hydroxycamptothecin (SN-38, an active metabolite of irinotecan hydrochloride) was purchased from LKT Laboratories, Inc. (St. Paul, MN, USA). Mitoxantrone dihydrochloride was obtained from Sigma-Aldrich Chemical Co. (St. Louis, MO, USA). 2-(4-Iodophenyl)-3-(4nitrophenyl)-5-(2,4-disulfophenyl)-2 $H$-tetrazolium, monosodium salt (WST-1) and 1-methoxy-5-methylphenazinium methylsulfate were acquired from Dojindo Laboratories (Kumamoto, Japan).

Cells and cell culture. The human acute promyelocytic leukemia cell line HL60 (JCRB0085; Japanese Collection of Research Bioresources Cell Bank, National Institutes of Biomedical Innovation, Health and Nutrition, Osaka, Japan), which is used as a model of leukemia cells, was routinely cultured in medium consisting of RPMI1640 (Sigma-Aldrich) supplemented with 10\% heat-inactivated fetal bovine serum $\left(\mathrm{Gibco}^{\mathrm{TM}}\right.$, Thermo Fisher Scientific K.K., Tokyo, Japan) and $50 \mathrm{U} / \mathrm{ml}$ penicillin-50 $\mu \mathrm{g} / \mathrm{ml}$ streptomycin (Invitrogen $^{\mathrm{TM}}$, Thermo Fisher Scientific). HL60 cells were grown in culture flasks in humidified air with $5 \% \mathrm{CO}_{2}$ at $37^{\circ} \mathrm{C}$, and subcultured every 3 or 4 days.

The human cervical carcinoma cell line HeLa, selected as a model of cancer cells, was maintained in Dulbecco's modified Eagle's medium (DMEM, Sigma-Aldrich) supplemented with $10 \%$ heat-inactivated fetal bovine serum $\left(\mathrm{Gibco}^{\mathrm{TM}}\right)$ and $100 \mathrm{mg} / \mathrm{l}$ of kanamycin sulfate (Invitrogen $\left.{ }^{\mathrm{TM}}\right)(22,23)$. Cells were seeded into culture flasks, grown in a humidified atmosphere of $5 \% \mathrm{CO}_{2}-95 \%$ air at $37^{\circ} \mathrm{C}$, and subcultured with $0.05 \%$ trypsin- $0.02 \%$ ethylenediaminetetra-acetic acid (Invitrogen ${ }^{\mathrm{TM}}$ ). HeLa/TXL and HeLa/SN100 cells were previously established by continuous exposure to paclitaxel or SN-38, respectively $(22,23)$. HeLa/TXL and HeLa/SN100 cells were maintained in a manner similar to HeLa cells, except that DMEM contained $20 \mathrm{nM}$ of paclitaxel and $100 \mathrm{nM}$ of SN-38, respectively.

Establishment of venetoclax-resistant HL60 sublines. Briefly, HL60 cells were cultured in RPMI1640 medium containing $1 \mu \mathrm{M}$ venetoclax, as a sub-growth-inhibition concentration, for approximately 3 months. The cells stably growing in the presence of $1 \mu \mathrm{M}$ venetoclax were isolated and then cloned by a colonyformation method using a RPMI1640 medium containing $0.75 \%$ methylcellulose. In the preliminary experiments, the characteristics of several clones were examined, and the selected clone was designated as HL60/VEN. HL60/VEN cells were maintained in a manner similar to HL60 cells, except that the RPMI1640 medium contained $1 \mu \mathrm{M}$ venetoclax.
Cell proliferation assay. The effects of the test drugs on the cell proliferation of HL60 and HL60/VEN cells were evaluated by a WST-1 assay $(22,23)$. The cells $\left(2.5 \times 10^{3} /\right.$ well $)$ were seeded into a 96-well plate in $50 \mu \mathrm{l}$ of RPMI1640 medium without any drugs. After $24 \mathrm{~h}$ of pre-culture, each well was supplemented with $50 \mu \mathrm{l}$ medium containing different concentrations of the test drugs. After incubation for $72 \mathrm{~h}$ at $37^{\circ} \mathrm{C}, 10 \mu \mathrm{l}$ of WST-1 solution was added to the wells of the 96-well plates; $5 \mathrm{~h}$ later, the absorbance was measured at $450 \mathrm{~nm}$ with a reference wavelength of $620 \mathrm{~nm}$ using a Spectra Fluor microplate reader (Tecan Switzerland, Switzerland).

HeLa and its resistant cell line were seeded $\left(1 \times 10^{3} /\right.$ well $)$ into 96 well plates in $100 \mu \mathrm{l}$ of DMEM without any drugs. After a $24 \mathrm{~h}$ preculture, the medium was replaced with medium containing a test drug at different concentrations. After incubation for $72 \mathrm{~h}$ at $37^{\circ} \mathrm{C}$, DMEM was replaced with $110 \mu \mathrm{l}$ of medium containing WST-1 reagent $(10 \mu \mathrm{l}$ of WST-1 $+100 \mu \mathrm{l}$ of DMEM); $3 \mathrm{~h}$ later, the absorbance was determined as mentioned above. The $50 \%$ growthinhibitory concentration $\left(\mathrm{IC}_{50}\right)$ of the test drugs was estimated according to the sigmoid inhibitory effect model, $\mathrm{E}=\mathrm{E}_{\max } \times[1-$ $\left.\mathrm{C} \gamma /\left(\mathrm{C} \gamma+\mathrm{IC}_{50} \gamma\right)\right]$, using the nonlinear least-squares fitting method (Solver, Microsoft ${ }^{\circledR}$ Excel), in which $\mathrm{E}$ and $\mathrm{E}_{\max }$ represent the surviving fraction (\% of control) and its maximum, respectively; $\mathrm{C}$ and $\gamma$ represent the concentration in the medium and the sigmoidicity factor, respectively $(22,23)$.

5-Bromo-2'-deoxyuridine (BrdU) incorporation assay. DNA synthesis as an index for growth activity was assessed using the BrdU incorporation assay (Cell Proliferation ELISA, BrdU (colorimetric); Roche Diagnostics K.K., Tokyo, Japan). Cells $\left(5 \times 10^{3} /\right.$ well) were seeded into 96-well plates in $50 \mu \mathrm{l}$ of RPMI1640 medium without any drugs. After a $24 \mathrm{~h}$ pre-culture, $50 \mu \mathrm{l}$ of medium containing 0.08 to $20 \mu \mathrm{M}$ of venetoclax was added to each well, resulting in a final concentration of 0.04 to $10 \mu \mathrm{M}$ of venetoclax; the cells were further incubated for $72 \mathrm{~h}$. According to the manufacturer's instructions, the incorporation of BrdU was conducted $24 \mathrm{~h}$ after treatment with venetoclax. After removing the BrdU labeling reagents, cells were fixed, and the BrdU-labeled DNA was denatured. Samples were incubated for 90 min with an anti-BrdU monoclonal antibody conjugated with peroxidase, and the substrates for peroxidase were added to the samples. The absorbance of the samples was measured at $360 \mathrm{~nm}$ with a reference wavelength of $450 \mathrm{~nm}$ using a Spectra Fluor microplate reader (Tecan).

Flow cytometry. HL60 and HL60/VEN cells were treated with 5 or 10 $\mu \mathrm{M}$ venetoclax for $48 \mathrm{~h}$. Thereafter, cells were harvested and labeled with fluorescein isothiocyanate (FITC)-conjugated annexin V (BioLegend, San Diego, CA, USA) and propidium iodide (PI, 50 $\mu \mathrm{g} / \mathrm{ml}$; FUJIFILM Wako) for detection of early and late apoptosis. FITC and PI fluorescence signals were detected using a FACSCalibur $^{\mathrm{TM}}$ instrument (Becton Dickinson, Franklin Lakes, NJ, USA) $(24,25)$. Data were analyzed using CELLQuest Ver. 3.3 (Becton Dickinson) software. At least 10,000 cells were used in the analysis.

DNA fragmentation assay. As a common feature of apoptosis, DNA fragmentation after exposure to venetoclax was examined (26). HL60 and HL60/VEN cells were seeded onto plastic culture dishes $(60 \mathrm{~mm}$ in diameter) in $5 \mathrm{ml}$ of RPMI1640 medium. After cells were precultured for $24 \mathrm{~h}, 1,5,10$, and $20 \mu \mathrm{M}$ venetoclax was added to the cells which were incubated for a further $24 \mathrm{~h}$ in a humidified atmosphere containing $5 \% \mathrm{CO}_{2}$ at $37^{\circ} \mathrm{C}$. Cells were then collected, 
Table I. Sequences of oligonucleotide primers used in quantitative reverse transcription-polymerase chain reaction in this study.

\begin{tabular}{llcc}
\hline Gene symbol & Encoded protein & Forward, 5'-3' orientation & Reverse, $5^{\prime}$-3' orientation \\
\hline$A C T B$ & $\beta$-Actin & TCATGAAGTGTGACGTGGACATC & TGCATCCTGTCGGCAATG \\
$B A K 1$ & BCL2 antagonist/killer 1 & ATGGTCACCTTACCTCTGCAA & TCATAGCGTCGGTTGATGTCG \\
$B A X$ & BCL2-associated X, apoptosis regulator & TGGCAGCTGACATGTTTCTGAC & TCACCCAACCACCCTGGTCTT \\
$B C L 2$ & BCL2 apoptosis regulator & TCGCCCTGTGGATGACTGA & CAGAGACAGCCAGGAGAAATCA \\
$B C L 2 L 12$ & BCL2-like 12 & CCCTCGGCCTTGCTCTCT & TCCGCAGTATGGCTTCCTTC \\
$B C L 2 L 1$ & BCL2-like 1 & GCTTGGATGGCCACTTACCT & TCAGGAACCAGCGGTTGAAG \\
$B C L 2 A 1$ & BCL2 related protein A1 & ACACAGGAGAATGGATAAGGCA & TGGTCAACAGTATTGCTTCAGGA \\
$M C L 1$ & MCL1 apoptosis regulator, BCL2 family member & GGACAAAACGGGACTGGCTA & CAGCAGCACATTCCTGATGC \\
$A B C B 1$ & ATP-binding cassette subfamily B member 1 & TTCCTTCACCCAGGCAATG & ATGAGTTATGTGCCACCAAGTAG \\
$A B C C 1$ & ATP-binding cassette subfamily C member 1 & CAGTGACCTCTGGTCCTTAAACAA & TTGGCGCATTCCTTCTTCC \\
$A B C C 2$ & ATP-binding cassette subfamily C member 2 & ACTTGTGACATCGGTAGCATGGA & AAGAGGCAGTTGTGAGGGATGA \\
$A B C G 2$ & ATP-binding cassette subfamily G member 2 & TGACGGTGAGAGAAAACTTAC & TGCCACTTTATCCAGACCT \\
\hline
\end{tabular}

and RNase and protein digestion enzyme solution were added using an Apoptosis Ladder Detection kit (FUJIFILM Wako). The DNA extracted from cells was electrophoresed on a $2 \%$ agarose gel. After electrophoresis, the agarose gels were stained with SYBR ${ }^{\circledR}$ green, and DNA fragmentation was detected by UV transillumination.

Quantitative reverse transcription-polymerase chain reaction $(R T$ $P C R)$. After cells were precultured for $48 \mathrm{~h}$, total RNA was extracted from the cells with a GenElute ${ }^{\mathrm{TM}}$ Mammalian Total RNA Miniprep kit (Sigma-Aldrich). The mRNA expression levels of BCL2 antagonist/killer 1 (BAK1), BCL2-associated $\mathrm{X}$ apoptosis regulator $(B A X), B C L 2$, BCL2-like 1 (BCL2L1), BCL2-like 12 (BCL2L12), BCL2-related protein A1 (BCL2A1), MCL1 apoptosis regulator, BCL2 family member ( $M C L 1)$, ATP binding cassette subfamily B member $1(A B C B 1), A B C C 1, A B C C 2$ and $A B C G 2$ were measured by RT-PCR (27). Total RNA (500 ng) was used for RT reaction with a PrimeScript $^{\mathrm{TM}}$ RT reagent kit (Takara Bio Inc., Shiga, Japan) and a thermal cycler (i-Cycler; Bio-Rad Laboratories, Hercules, CA, USA). The RT reaction was conducted in $20 \mu \mathrm{l}$ of reaction buffer at $37^{\circ} \mathrm{C}$ for $15 \mathrm{~min}$, and terminated by heating at $85^{\circ} \mathrm{C}$ for $5 \mathrm{~s}$ followed by cooling at $4^{\circ} \mathrm{C}$. Real-time PCR was performed with a 7500 Fast RealTime PCR system (Applied Biosystems, Waltham, MA, USA) and SYBR Premix Ex Taq ${ }^{\mathrm{TM}}$ (Takara Bio). $\beta$-Actin $(A C T B)$ was used as an internal standard. The primers were synthesized by GeneDesign, Inc. (Ibaraki, Japan) and their sequences are presented in Table I. To compare the relative target mRNA levels, the comparative threshold cycle method was used, as previously described (27).

Statistical analysis. Comparisons between two groups or among three groups were performed with Student's unpaired $t$-test or a nonrepeated one-way analysis of variance followed by the Dunnett test, respectively, using JMP ${ }^{\circledR}$ Pro 15.2.0. (SAS Institute Japan Ltd., Tokyo, Japan). A $p$-value of less than 0.05 (two-tailed) was considered significant.

\section{Results}

Sensitivity to venetoclax. The $\mathrm{IC}_{50}$ values for venetoclax in HL60 and HL60/VEN cells are indicated in Table II. Treatment with venetoclax for $72 \mathrm{~h}$ caused growth inhibition of HL60 and HL60/VEN cells, with IC $_{50}$ values of $2.31 \mu \mathrm{M}$ and $6.26 \mu \mathrm{M}$, respectively. Such findings indicate a 2.71-fold greater resistance of HL60/VEN cells relative to HL60 cells. The effects of venetoclax on the DNA incorporation of BrdU into HL60 and HL60/VEN cells were examined (Figure 1A). The incorporation of BrdU into DNA in HL60 cells was unchanged up to $0.625 \mu \mathrm{M}$ venetoclax, whilst significant suppression was observed at 2.5 and $10 \mu \mathrm{M}$ of venetoclax. However, BrdU incorporation in HL60/VEN cells was maintained up to $2.5 \mu \mathrm{M}$ venetoclax, although a significant increase was noted at $0.625 \mu \mathrm{M}$ venetoclax, and significant inhibition was observed at $10 \mu \mathrm{M}$ venetoclax.

DNA fragmentation and apoptosis induction by venetoclax. Fragmentation of DNA by exposure to venetoclax was examined in HL60 and HL60/VEN cells (Figure 1B). DNA fragmentation was observed in both cell lines treated with venetoclax, but the onset of DNA fragmentation in HL60/VEN cells occurred at a higher concentration compared with that in HL60 cells.

The induction of apoptosis in HL60 and HL60/VEN cells was measured by FITC-annexin V/PI staining after treatment with venetoclax (Figure 1C and D). The percentage of early and late apoptosis after $24 \mathrm{~h}$ of venetoclax treatment concentration-dependently increased in HL60 cells. Although the numbers of early and late apoptotic cells in HL60/VEN cells also increased in a venetoclax concentration-dependent manner, their fraction was significantly lower in HL60/VEN cells than in HL60 cells.

Expression profiles of apoptotic regulating factors. The mRNA expression levels of apoptosis-related factors were examined by real-time quantitative RT-PCR. The mRNA level of BCL2, an apoptosis-inhibitory factor, was significantly lower in HL60/VEN cells than HL60 cells, resulting in an expression 
Table II. Sensitivity of HL60 and HL60/VEN cells to different types of anticancer drugs. Each 50\% growth-inhibitory concentration $\left(I C_{50}\right)$ value represents the mean \pm standard deviation $(n=8)$.

\begin{tabular}{lcccc}
\hline \multirow{2}{*}{ Drug } & \multirow{2}{*}{ Unit } & \multicolumn{2}{c}{$\mathrm{IC}_{50}$ value } & \multirow{2}{*}{$\mathrm{RR}$} \\
\cline { 3 - 4 } & & $\mathrm{HL60}$ & $\mathrm{HL60/VEN}$ & \\
\hline Venetoclax & $\mu \mathrm{M}$ & $2.31 \pm 0.12$ & $6.26 \pm 0.81^{* *}$ & 2.71 \\
Vincristine & $\mathrm{nM}$ & $11.2 \pm 2.65$ & $23.0 \pm 6.02^{* *}$ & 2.05 \\
Vinblastine & $\mathrm{nM}$ & $45.1 \pm 4.71$ & $100 \pm 9.13^{* *}$ & 2.10 \\
Paclitaxel & $\mathrm{nM}$ & $0.095 \pm 0.019$ & $0.272 \pm 0.090^{* *}$ & 2.70 \\
Etoposide & $\mu \mathrm{M}$ & $0.293 \pm 0.015$ & $1.53 \pm 0.32^{* *}$ & 5.28 \\
Doxorubicin & $\mathrm{nM}$ & $20.3 \pm 1.21$ & $122 \pm 18.2 * *$ & 5.99 \\
SN-38 & $\mathrm{nM}$ & $4.21 \pm 0.32$ & $4.93 \pm 0.73$ & 1.17 \\
Mitoxantrone & $\mathrm{nM}$ & $15.6 \pm 0.69$ & $23.8 \pm 1.20^{* *}$ & 1.52 \\
5-Fluorouracil & $\mu \mathrm{M}$ & $37.1 \pm 6.58$ & $46.6 \pm 9.10$ & 1.25 \\
Cisplatin & $\mu \mathrm{M}$ & $3.04 \pm 0.27$ & $3.06 \pm 0.32$ & 1.00 \\
Carboplatin & $\mu \mathrm{M}$ & $17.1 \pm 1.39$ & $27.3 \pm 2.22 * *$ & 1.60 \\
\hline
\end{tabular}

RR: Relative resistance, calculated as the $\mathrm{IC}_{50}$ value for HL60/VEN cells divided by the value for HL60 cells. ${ }^{* *}$ Significantly different at $p<0.01$ from HL60 cells (unpaired Student's $t$-test).

ratio of 0.21 relative to that of HL60 cells (Figure 2A and C). However, the mRNA levels of BCL2Al and MCL1, which are also apoptosis-inhibitory factors, were significantly higher in HL60/VEN cells than HL60 cells, resulting in expression ratios of 1.79 and 1.33 , respectively. There was no difference in the expression of BCL2L1 and BCL2L12 mRNAs between cell lines. In addition, there was no significant difference in the mRNA expression of the apoptosis-promoting factors $B A K 1$ and $B A X$ (Figure 2B and C).

Sensitivity to different types of anticancer drugs. Table II summarizes the $\mathrm{IC}_{50}$ values for different types of anticancer drugs against HL60 and HL60/VEN cells. The $\mathrm{IC}_{50}$ values for vincristine, vinblastine, paclitaxel, etoposide, and doxorubicin, which are substrates for ABCB1, were significantly higher for HL60/VEN cells than HL60 cells. No significant difference in the sensitivity to SN-38, an active metabolite of irinotecan hydrochloride and substrate of ABCG2, was found between HL60 and HL60/VEN cells. However, the sensitivity to mitoxantrone, a substrate for ABCG2, was significantly reduced in HL60/VEN cells. There were no differences in sensitivity to 5-fluorouracil and cisplatin in either cell line; however, sensitivity to carboplatin was significantly lower in HL60/VEN cells.

Expression profiles of selected ABC transporters. Figure 3 shows the expression level of the representative $A B C$ transporters, which are involved in resistance to various types of anticancer drugs. The expression level of $A B C B 1$ mRNA in HL60/VEN cells was significantly higher than that in HL60 cells, and that of $A B C G 2$ mRNA tended to be increased in
HL60/VEN cells. The levels of $A B C C 1$ and $A B C C 2$ mRNAs were significantly reduced in HL60/VEN cells.

Participation of $A B C B 1$ in the resistance to venetoclax. To clarify the participation of $\mathrm{ABCB} 1$ in the resistance of HL60/VEN cells to venetoclax, the effect of cyclosporine A, an inhibitor of $\mathrm{ABCB} 1$, on sensitivity to venetoclax was determined (Table IV). The $\mathrm{IC}_{50}$ values for venetoclax against HL60 and HL60/VEN cells in the presence of $1 \mu \mathrm{M}$ cyclosporine $\mathrm{A}$ were comparable to the respective $\mathrm{IC}_{50}$ values in the absence of cyclosporine A.

Sensitivity to venetoclax in $A B C B 1-$ or $A B C G 2-$ overexpressing cells. The sensitivity to venetoclax was examined in the ABCB1-overexpressing HeLa/TXL and ABCG2-overexpresssing HeLa/SN100 cells, which were previously established via continuous exposure to paclitaxel or $\mathrm{SN}-38(22,23)$. The $\mathrm{IC}_{50}$ values for venetoclax were comparable between HeLa and the ABCB1-overexpressing HeLa/TXL cells (Table III). The $\mathrm{IC}_{50}$ value for venetoclax against ABCG2-overexpressing $\mathrm{HeLa} / \mathrm{SN} 100$ cells was significantly lower than those in HeLa cells.

\section{Discussion}

Herein, venetoclax-resistant cells were newly established through the continuous exposure of HL60 cells, a leukemia cell model, to $1 \mu \mathrm{M}$ venetoclax (clinically achievable plasma concentration); these cells were designated as HL60/VEN cells. Compared with HL60 cells, HL60/VEN cells showed approximately 3 -fold resistance to venetoclax in the cell growth-inhibition assay (Table II). BrdU incorporation was maintained at a higher concentration of venetoclax in HL60/VEN cells than in HL60 cells (Figure 1A), implying that HL60/VEN cells exhibited resistance to venetoclax from the viewpoint of their ability to synthesize DNA. Based on these findings, the established HL60/VEN cells were characterized as venetoclax-resistant, and were demonstrated to be a useful tool for identifying the potential mechanisms of resistance.

The induction of apoptosis by venetoclax was lower in HL60/VEN cells than HL60 cells based on annexin V staining and DNA fragmentation assay (Figure 1B-D). These findings indicate a change in the apoptotic pathway of HL60/VEN cells, suggesting that this alteration was at least involved in the mechanism of resistance to venetoclax in HL60/VEN cells. The mRNA expression levels of the BCL2 family proteins were examined by real-time RT-PCR. The mRNA expression of BCL2 was significantly lower in HL60/VEN cells, whereas that of $B C L 2 A 1$ and MCL1 increased significantly (Figure 2A). Some studies reported that a low or lack of expression of BCL2 induced high resistance to venetoclax, consistent with the characteristics of HL60/VEN cells, although the examined cell lines differed from those used in this study (28-30). Previous 
A

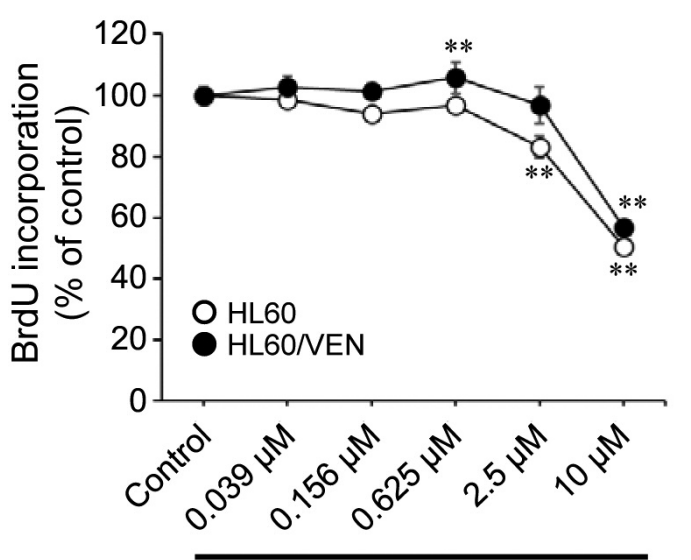

Venetoclax conc.
B

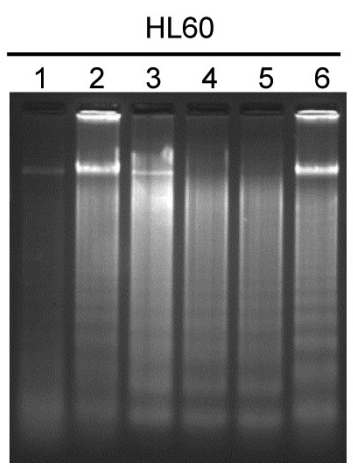

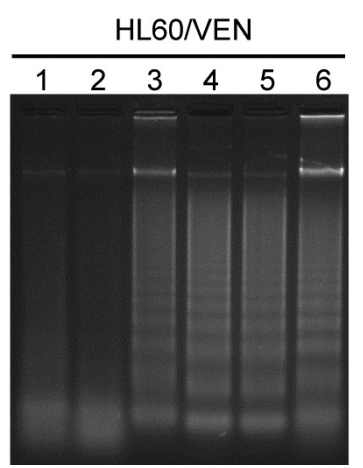

C
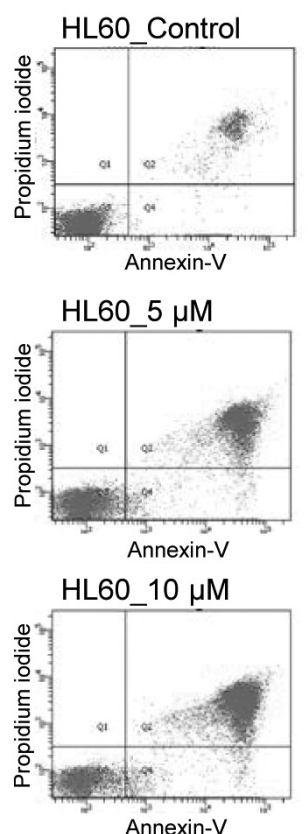

HL60/VEN Control
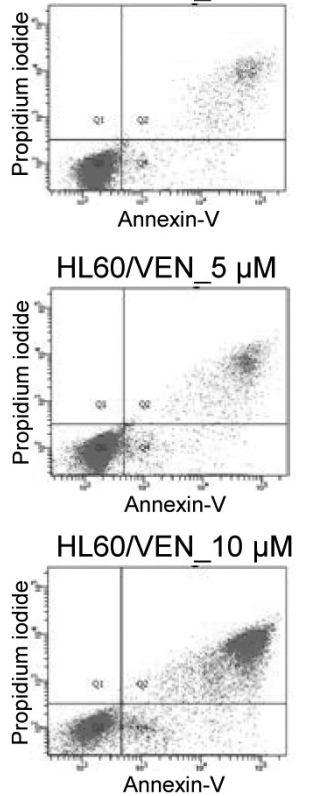

D

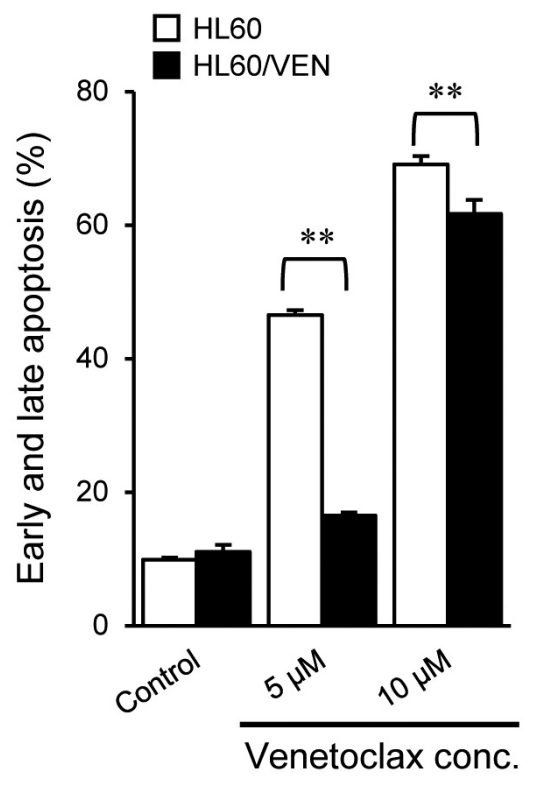

Figure 1. Molecular characteristics of HL60 and HL/VEN cells. A: Incorporation of 5-bromo-2'-deoxyuridine (BrdU) into DNA by HL60 and HL60/VEN cells. Cells were treated with venetoclax at 0.04 to $10 \mu \mathrm{M}$ for $72 \mathrm{~h}$, and the incorporation of BrdU was conducted for $24 \mathrm{~h}$. BrdUlabeled DNA were determined colorimetrically using a cell proliferation enzyme-linked immunosorbent assay. Each bar shows the mean \pm standard deviation $(n=4)$. $* *$ Significantly different at $p<0.01$ from the respective control (non-repeated analysis of variance followed by Dunnett test). B: Electropherograms of DNA fragmentation after exposure to venetoclax in HL60 and HL60/VEN cells. HL60 and HL60/VEN cells were treated with 1, 5, 10, and $20 \mu \mathrm{M}$ venetoclax for $24 \mathrm{~h}$. The extracted DNA from cells was electrophoresed by $2 \%$ agarose gel, and were stained by $S Y B R^{\circledR}$ green. The lane identifications were as follows. Lane 1: Control (no treatment with drug); lane 2: $1 \mu M$ venetoclax; lane 3: $5 \mu \mathrm{M}$ venetoclax; lane 4: $10 \mu \mathrm{M}$ venetoclax treatment; Lane 5: $20 \mu \mathrm{M}$ venetoclax; lane 6: Positive control (treatment with $1 \mu \mathrm{M}$ cisplatin). C; Flow cytometry-based detection of apoptosis by concurrent staining with annexin V-fluorescein isothiocyanate (FITC) and propidium iodide (PI). HL60 and HL60/VEN cells were treated with 5 or $10 \mu M$ venetoclax for 48 h. Cells were labeled with FITC-conjugated annexin V and PI for early and late apoptosis detection. Cells were regarded as follows: Stained with neither annexin V nor PI: live cells (Q3); stained only with annexin V: early apoptotic cells (Q4); and stained with both annexin V and PI: late apoptotic cells (Q2). D: Quantification of flow cytometric data showing the proportion of apoptotic cells (early plus late apoptosis). **Significantly different at $p<0.01$ from the respective HL60 cells (unpaired Student's t-test). 


\section{A Apoptosis-inhibitory factors}
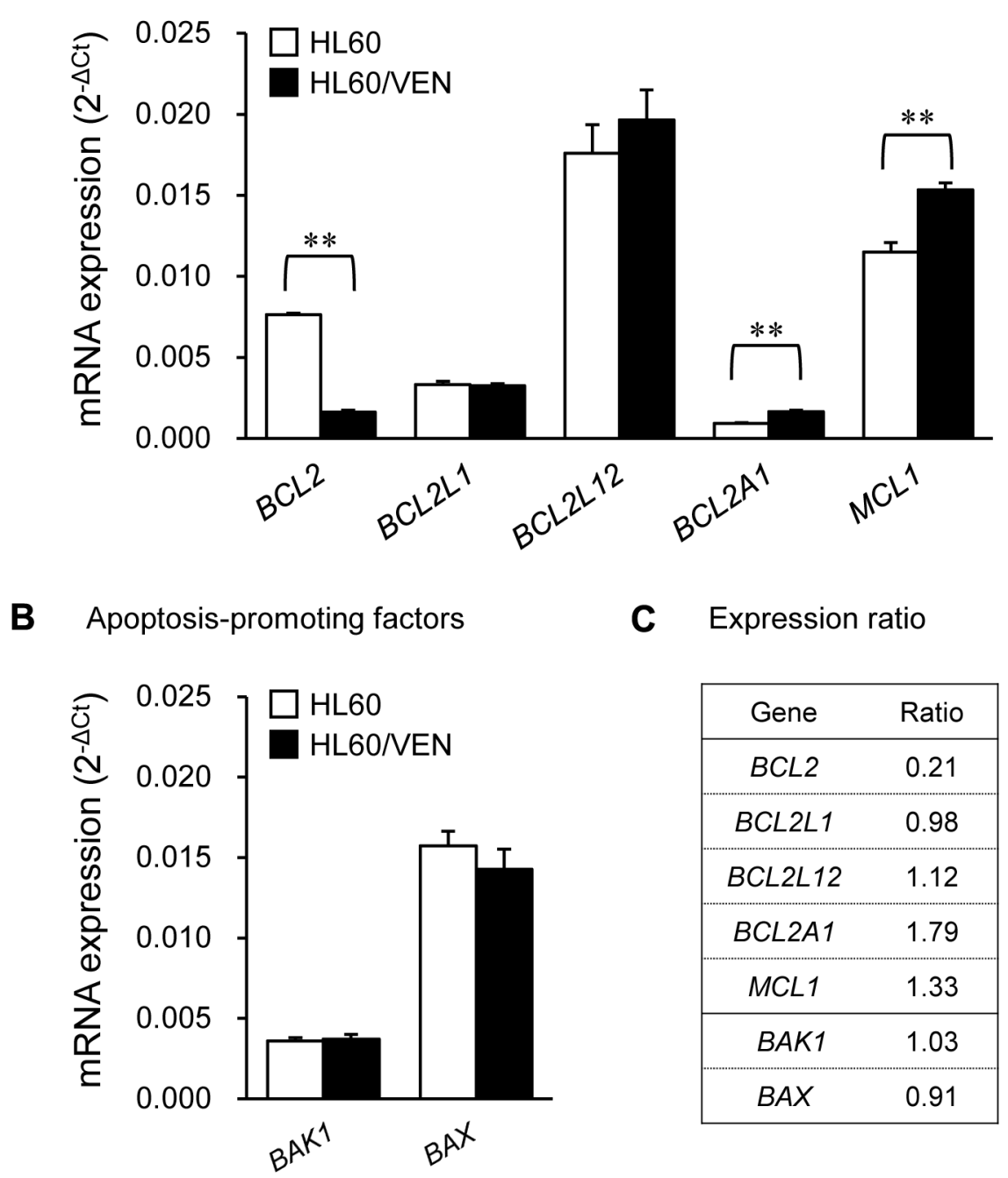

C Expression ratio

Figure 2. mRNA expression profile of apoptosis-regulating factors in HL60 and HL60/VEN cells. Total RNA was extracted from HL60 and HL60/VEN cells using a spin column method. The mRNA expressions of target gene and $\beta$-actin, as an internal standard, were evaluated by real-time reverse transcription-polymerase chain reaction using a SYBR ${ }^{\circledR}$ green. To compare the relative expression of target mRNA levels, the comparative threshold cycle method was used. A: Expression of apoptosis-inhibitory factors BCL2 apoptosis regulator (BCL2), BCL2-like 1 (BCL2L1), BCL2-like 12 (BCL2L12), BCL2-related protein A1 (BCL2A1) and MCL1 apoptosis regulator, BCL2 family member (MCL1). B: Expression of apoptosis-promoting factors BCL2 antagonist/killer 1 (BAK1) and BCL2-associated $X$ apoptosis regulator (BAX). C: The relative expression ratio was calculated as the expression level $\left(2^{-\triangle C t}\right)$ in HL60/VEN cells divided by that in the respective HL60 cells. Each bar represents the mean \pm standard deviation $(n=4)$. **Significantly different at $p<0.01$ from the respective HL60 cells (unpaired Student's t-test).

reports also revealed a high expression of MCL1 in venetoclaxresistant cells, which is similar to that found in HL60/VEN cells. Esteve-Arenys et al. $(31,32)$ reported that the accumulation of BCL2-related protein A1 is a major mechanism involved in acquired resistance to venetoclax; findings of the present study were comparable with this. Recently, Haselager et al. (33) demonstrated that BCL2-like 1 (BCL2L1) was also important in venetoclax resistance in chronic lymphocytic leukemia cells; however, the mRNA expression of $B C L 2 L 1$ was found to be comparable between HL60 and HL60/VEN cells in this study (Figure 2A). Such findings suggest complicated changes in expression of BCL2 family members owing to venetoclax resistance. Collectively, our findings suggest that HL60/VEN cells had the typical characteristics of venetoclax resistance. Investigating other potent mechanisms would thus be meaningful for reversing this resistance. 
A

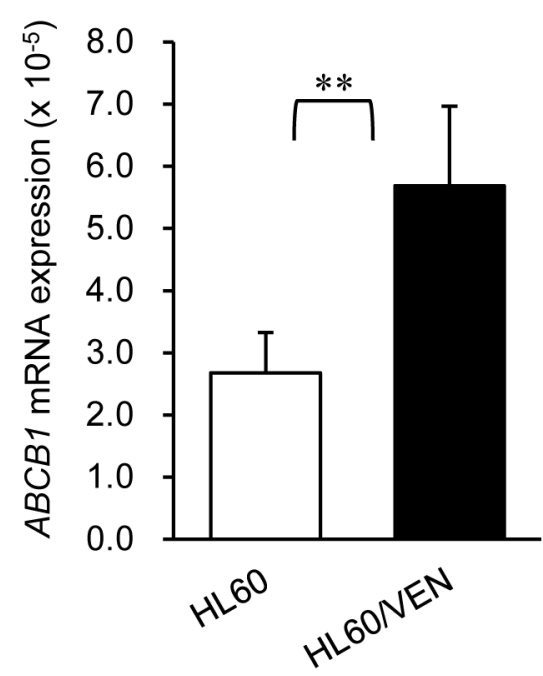

C

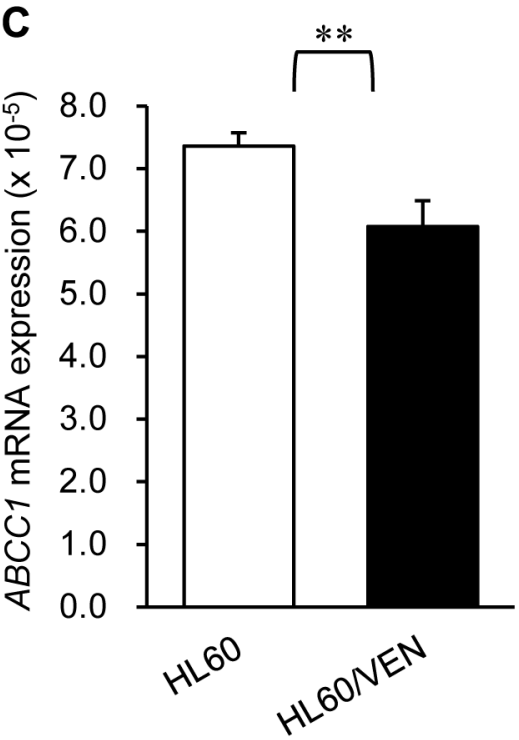

B

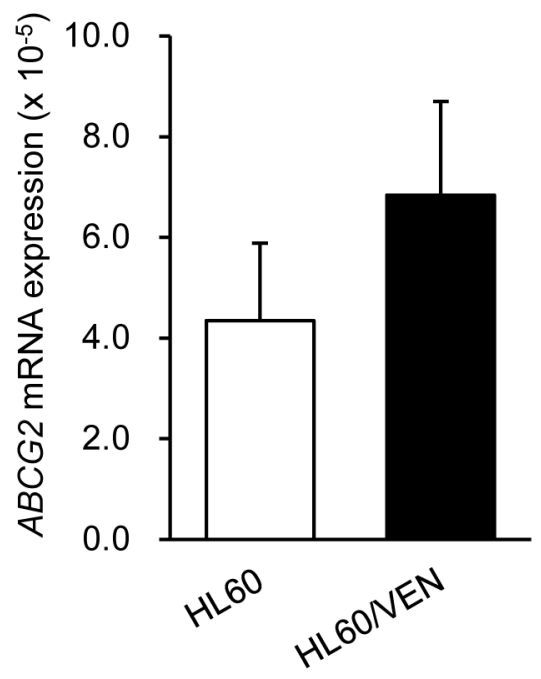

D

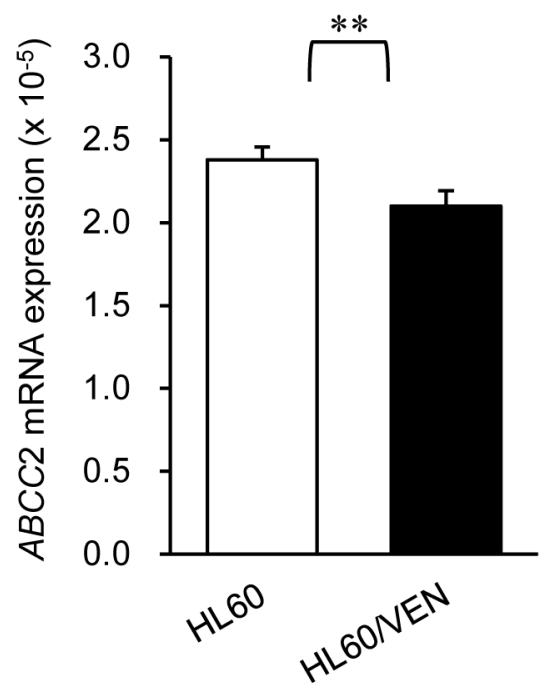

Figure 3. mRNA expression profiles of ATP-binding cassette subfamily B member 1 (ABCB1) (A), ABCG2 (B), ABCC1 (C), and ABCC2 (D) in HL60 and HL60/VEN cells. Total RNA was extracted from HL60 and HL60/VEN cells using a spin column method. The mRNA expressions of target gene and $\beta$-actin, as an internal standard, were evaluated by real-time reverse transcription-polymerase chain reaction using a SYBR ${ }^{\circledR}$ green. To compare the relative expression of target $m R N A$, the comparative threshold cycle method was used. Each bar represents the mean \pm standard deviation $(n=4)$. **Significantly different at $p<0.01$ from the respective HL60 cells (unpaired Student's t-test).

Interestingly, HL60/VEN cells showed diverse sensitivity to cytotoxic anticancer drugs, in particular, resistance to ABCB1 substrates (Table II). This resistance is supported by the up-regulation of $A B C B 1 \mathrm{mRNA}$ in HL60/VEN cells compared with HL60 cells (Figure 3). However, sensitivity to venetoclax in HL60/VEN cells was not restored by cyclosporine $\mathrm{A}$, a representative $\mathrm{ABCB} 1$ inhibitor, nor did it change the $\mathrm{IC}_{50}$ against HL60 cells (Table IV). Moreover,
ABCB1-overexpressing HeLa cells, established by continuous exposure to paclitaxel (22), showed comparable sensitivity to venetoclax relative to HeLa cells (Table III). These findings suggest that up-regulation of $A B C B I$ does not contribute to venetoclax resistance in HL60/VEN cells but elicits a cross-resistance to ABCB 1 substrates. The lack of contribution of $\mathrm{ABCB} 1$ to venetoclax resistance may be explained by the lower binding affinity of venetoclax for 
Table III. Sensitivity to venetoclax in ATP-binding cassette subfamily B member 1 (ABCB1)-overexpressing (HeLa/TXL) and ABCG2overexpressing (HeLa/SN1O0) cells. Each value for the 50\% growthinhibitory concentration $\left(I_{50}\right)$ represents the mean \pm standard deviation $(n=8$ or 16$)$.

\begin{tabular}{lcc}
\hline Cell line & $\mathrm{IC}_{50}$ value $(\mu \mathrm{M})$ & $\mathrm{RR}$ \\
\hline HeLa & $19.7 \pm 3.82$ & - \\
HeLa/TXL & $19.4 \pm 3.26$ & 0.98 \\
HeLa/SN100 & $16.1 \pm 1.49^{*}$ & 0.82 \\
\hline
\end{tabular}

RR: Relative resistance, calculated as the $\mathrm{IC}_{50}$ value for $\mathrm{HeLa} / \mathrm{TXL}$ or $\mathrm{HeLa} / \mathrm{SN} 100$ cells divided by that for HeLa cells. *Significantly different at $p<0.05$ from HeLa cells (non-repeated analysis of variance followed by Dunnett test).

Table IV. Effects of cyclosporine A (CyA) on the sensitivity to venetoclax of HL60 and HL60/VEN cells. Each value for the 50\% growthinhibitory concentration $\left(I_{50}\right)$ represents the mean \pm standard deviation $(n=8)$.

\begin{tabular}{lccc}
\hline Cells & \multicolumn{2}{c}{$\mathrm{IC}_{50}$ value $(\mu \mathrm{M})$} & RS \\
\cline { 2 - 3 } & Control & $+1 \mu \mathrm{M} \mathrm{CyA}$ & \\
\hline HL60 & $2.86 \pm 0.19$ & $3.04 \pm 0.39$ & 0.94 \\
HL60/VEN & $7.33 \pm 1.16$ & $7.36 \pm 2.02$ & 1.00 \\
\hline
\end{tabular}

RS: Relative sensitivity, calculated as the $\mathrm{IC}_{50}$ value for the respective control divided by that for cells treated with $1 \mu \mathrm{M}$ CyA.

ABCB1 than other cytotoxic anticancer drugs, e.g., vincristine and doxorubicin. In fact, the $\mathrm{IC}_{50}$ values of ABCB1-overexpressing murine monocytic leukemia P388/dx cells for in the transport assays using calcein, which is a substrate for $\mathrm{ABCB} 1$, were approximately $30 \mu \mathrm{M}$ for venetoclax and approximately $2.9 \mu \mathrm{M}$ for verapamil, a representative ABCB1 inhibitor (34).

Of note, the induction of $A B C B 1$ mRNA by continuous exposure to venetoclax was observed in this study (Figure 3 ). However, Weiss et al. (34) reported the suppression of $A B C B 1$ mRNA expression by venetoclax in LS180 human colon carcinoma cells, which is the standard model for investigating pregnane $\mathrm{X}$ receptor-mediated induction. The reason for this difference remains unclear; however, other mechanisms, other than those mediated by pregnane $\mathrm{X}$ receptor, may contribute to the induction of $A B C B 1$ in HL60 cells. In any case, the variation in expression of $A B C B 1$ induced by venetoclax was suggested to be clinically meaningful and a problem, leading to ABCB1-mediated multidrug resistance or drug-drug interaction.

By employing ABCG2-overexpressing cancer cells, Wang et al. (35) found that venetoclax potentiated the efficacy of ABCG2 substrate drugs by blocking ABCG2 efflux function and inhibiting the ATPase activity of ABCG2. The researchers also demonstrated that the cytotoxicity of venetoclax was similar between ABCG2mediated resistant cells and their parental cells, and that venetoclax did not affect the protein expression of ABCG2. The mRNA expression of $A B C G 2$ tended to increase in HL60/VEN cells (Figure 3 ) and the $\mathrm{IC}_{50}$ value for mitoxantrone but not $\mathrm{SN}-38$, both of which are ABCG2 substrates, was significantly high in HL60/VEN cells (Table II). However, the ABCG2-overexpressing $\mathrm{HeLa} / \mathrm{SN} 100$ cells, established by continuous exposure to SN-38 (23), displayed higher sensitivity to venetoclax than its parental cell line (Table III), although the relative resistance was not large $(0.82)$. The reasons for these complicated findings remain unclear; however, the use of venetoclax against cells with ABCG2-mediated resistance may be advantageous. Unexpectedly, the mRNA expression levels of $A B C C 1$ and $A B C C 2$ were significantly lower in HL60/VEN cells compared with HL60 cells (Figure 3). The down-regulation of the ABCC family suggests the potential of venetoclax to reverse resistance mediated by $\mathrm{ABCC} 1$ or $\mathrm{ABCC} 2$; however, there has been no report on the effects of venetoclax on these substrates as far as we are aware.

In conclusion, the venetoclax-resistant HL60/VEN cells established here displayed up-regulation of the expression and function of ABCB1, in addition to an alteration in the BCL2 family-associated pathway and apoptosis induction. Of note, resistance to other anticancer drug substrates of $\mathrm{ABCB} 1$, in addition to venetoclax, was demonstrated in HL60/VEN cells, although the sensitivity to venetoclax was barely affected by ABCB1. These findings highlight the need to monitor crossresistance and drug-drug interaction caused by the induction of $\mathrm{ABCB} 1$ by venetoclax in cancer chemotherapy.

\section{Conflicts of Interest}

The Authors declare that no conflicts of interest exist.

\section{Authors' Contributions}

Yuko Nakayama: Investigation, data curation, visualization and original draft preparation. Kohji Takara: Data curation, reviewing, editing and supervision. Tetsuya Minegaki: Investigation and data curation. Kazuhiro Yamamoto: Visualization and data curation. Tomohiro Omura: Reviewing and editing. Ikuko Yano: Reviewing, editing and supervision.

\section{Acknowledgements}

The Authors would like to thank Editage (www.editage.com) for English language editing. This work was supported in part by JSPS KAKENHI (JP26460246). 


\section{References}

1 Deeks ED: Venetoclax: First global approval. Drugs 76(9): 979987, 2016. PMID: 27260335. DOI: 10.1007/s40265-016-0596-x

2 Scott LJ: Venetoclax: A review in relapsed/refractory chronic lymphocytic leukemia. Target Oncol 14(5): 493-504, 2019. PMID: 31542870. DOI: 10.1007/s11523-019-00673-1

3 Kater AP, Seymour JF, Hillmen P, Eichhorst B, Langerak AW, Owen C, Verdugo M, Wu J, Punnoose EA, Jiang Y, Wang J, Boyer M, Humphrey K, Mobasher M and Kipps TJ: Fixed duration of venetoclax-rituximab in relapsed/refractory chronic lymphocytic leukemia eradicates minimal residual disease and prolongs survival: Post-treatment follow-up of the MURANO Phase III study. J Clin Oncol 37(4): 269-277, 2019. PMID: 30523712. DOI: $10.1200 / \mathrm{JCO} .18 .01580$

4 Hallek M: Chronic lymphocytic leukemia: 2020 update on diagnosis, risk stratification and treatment. Am J Hematol 94(11): 1266-1287, 2019. PMID: 31364186. DOI: 10.1002/ ajh. 25595

5 Roberts AW, Davids MS, Pagel JM, Kahl BS, Puvvada SD, Gerecitano JF, Kipps TJ, Anderson MA, Brown JR, Gressick L, Wong S, Dunbar M, Zhu M, Desai MB, Cerri E, Heitner Enschede S, Humerickhouse RA, Wierda WG and Seymour JF: Targeting BCL2 with venetoclax in relapsed chronic lymphocytic leukemia. N Engl J Med 374(4): 311-322, 2016. PMID: 26639348. DOI: 10.1056/NEJMoa1513257

6 Mihalyova J, Jelinek T, Growkova K, Hrdinka M, Simicek M and Hajek R: Venetoclax: A new wave in hematooncology. Exp Hematol 61: 10-25, 2018. PMID: 29477371. DOI: 10.1016/ j.exphem 2018.02 .002

7 Juárez-Salcedo LM, Desai V and Dalia S: Venetoclax: evidence to date and clinical potential. Drugs Context 8: 212574, 2019. PMID: 31645879. DOI: 10.7573/dic.212574

8 Siddiqui WA, Ahad A and Ahsan H: The mystery of BCL2 family: Bcl-2 proteins and apoptosis: an update. Arch Toxicol 89(3): 289-317, 2015. PMID: 25618543. DOI: 10.1007/s00204014-1448-7

9 Leahy DT, Mulcahy HE, O'Donoghue DP and Parfrey NA: bcl2 protein expression is associated with better prognosis in colorectal cancer. Histopathology 35(4): 360-367, 1999. PMID: 10564391. DOI: 10.1046/j.1365-2559.1999.00743.x

10 Hanahan D and Weinberg RA: The hallmarks of cancer. Cell 100(1): 57-70, 2000. PMID: 10647931. DOI: 10.1016/s00928674(00)81683-9

11 Delbridge AR, Grabow S, Strasser A and Vaux DL: Thirty years of BCL-2: translating cell death discoveries into novel cancer therapies. Nat Rev Cancer 16(2): 99-109, 2016. PMID: 26822577. DOI: $10.1038 / \mathrm{nrc} .2015 .17$

12 Anderson MA, Huang D and Roberts A: Targeting BCL2 for the treatment of lymphoid malignancies. Semin Hematol 51(3): 219227, 2014. PMID: 25048785. DOI: 10.1053/j.seminhematol. 2014.05.008

13 Valentin R, Grabow S and Davids MS: The rise of apoptosis: targeting apoptosis in hematologic malignancies. Blood 132(12): 1248-1264, 2018. PMID: 30012635. DOI: 10.1182/blood-201802-791350

14 Perini GF, Ribeiro GN, Pinto Neto JV, Campos LT and Hamerschlak N: BCL-2 as therapeutic target for hematological malignancies. J Hematol Oncol 11(1): 65, 2018. PMID: 29747654. DOI: 10.1186/s13045-018-0608-2
15 Anderson MA, Deng J, Seymour JF, Tam C, Kim SY, Fein J, Yu L, Brown JR, Westerman D, Si EG, Majewski IJ, Segal D, Heitner Enschede SL, Huang DC, Davids MS, Letai A and Roberts AW: The BCL2 selective inhibitor venetoclax induces rapid onset apoptosis of CLL cells in patients via a TP53independent mechanism. Blood 127(25): 3215-3224, 2016. PMID: 27069256. DOI: 10.1182/blood-2016-01-688796

16 Punnoose EA, Leverson JD, Peale F, Boghaert ER, Belmont LD, Tan N, Young A, Mitten M, Ingalla E, Darbonne WC, Oleksijew A, Tapang P, Yue P, Oeh J, Lee L, Maiga S, Fairbrother WJ, Amiot M, Souers AJ and Sampath D: Expression profile of BCL-2, BCL-XL, and MCL-1 predicts pharmacological response to the BCL-2 selective antagonist venetoclax in multiple myeloma models. Mol Cancer Ther 15(5): 1132-1144, 2016. PMID: 26939706. DOI: 10.1158/1535-7163.MCT-15-0730

17 Takara K, Sakaeda T and Okumura K: An update on overcoming MDR1-mediated multidrug resistance in cancer chemotherapy. Curr Pharm Des 12(3): 273-286, 2006. PMID: 16454744. DOI: $10.2174 / 138161206775201965$

18 Takara K, Sakaeda T and Okumura K: Carvedilol: a new candidate for reversal of MDR1/P-glycoprotein-mediated multidrug resistance. Anticancer Drugs 15(4): 303-309, 2004. PMID: 15057133. DOI: 10.1097/00001813-200404000-00001

19 Bose P, Gandhi V and Konopleva M: Pathways and mechanisms of venetoclax resistance. Leuk Lymphoma 58(9): 1-17, 2017. PMID: 28140720. DOI: 10.1080/10428194.2017.1283032

20 Tausch E, Close W, Dolnik A, Bloehdorn J, Chyla B, Bullinger L, Döhner H, Mertens D and Stilgenbauer S: Venetoclax resistance and acquired $B C L 2$ mutations in chronic lymphocytic leukemia. Haematologica 104(9): e434-e437, 2019. PMID: 31004028. DOI: 10.3324/haematol.2019.222588

21 Pei S, Pollyea DA, Gustafson A, Stevens BM, Minhajuddin M, Fu R, Riemondy KA, Gillen AE, Sheridan RM, Kim J, Costello JC, Amaya ML, Inguva A, Winters A, Ye H, Krug A, Jones CL, Adane B, Khan N, Ponder J, Schowinsky J, Abbott D, Hammes A, Myers JR, Ashton JM, Nemkov T, D’Alessandro A, Gutman JA, Ramsey HE, Savona MR, Smith CA and Jordan CT: Monocytic subclones confer resistance to venetoclax-based therapy in patients with acute myeloid leukemia. Cancer Discov 10(4): 536-551, 2020. PMID: 31974170. DOI: $10.1158 / 2159-8290 . C D-19-0710$

22 Takara K, Obata Y, Yoshikawa E, Kitada N, Sakaeda T, Ohnishi $\mathrm{N}$ and Yokoyama T: Molecular changes to HeLa cells on continuous exposure to cisplatin or paclitaxel. Cancer Chemother Pharmacol 58(6): 785-793, 2006. PMID: 16534613. DOI: $10.1007 / \mathrm{s} 00280-006-0226-5$

23 Takara K, Kitada N, Yoshikawa E, Yamamoto K, Horibe S, Sakaeda T, Nishiguchi K, Ohnishi N and Yokoyama T: Molecular changes to HeLa cells on continuous exposure to $\mathrm{SN}-38$, an active metabolite of irinotecan hydrochloride. Cancer Lett 278(1): 8896, 2009. PMID: 19201079. DOI: 10.1016/j.canlet.2008.12.033

24 Takara K, Yamamoto K, Matsubara M, Minegaki T, Takahashi $\mathrm{M}$, Yokoyama $\mathrm{T}$ and Okumura K: Effects of $\alpha$-adrenoceptor antagonists on ABCG2/BCRP-mediated resistance and transport. PLoS One 7(2): e30697, 2012. PMID: 22355323. DOI: 10.1371/ journal.pone.0030697

25 Miyamoto K, Minegaki T, Hirano S, Hayashi I, Tsujimoto M and Nishiguchi K: Olaparib potentiates anticancer drug cytotoxicity via 53BP1 in oesophageal squamous cell carcinoma cells. Anticancer Res 40(2): 813-823, 2020. PMID: 32014924. DOI: 10.21873/anticanres.14013 
26 Kitada N, Takara K, Itoh C, Minegaki T, Tsujimoto M, Sakaeda $\mathrm{T}$ and Yokoyama T: Comparative analysis of cell injury after exposure to antitumor platinum derivatives in kidney tubular epithelial cells. Chemotherapy 54(3): 217-223, 2008. PMID: 18560229. DOI: $10.1159 / 000140465$

27 Kitada N, Takara K, Minegaki T, Itoh C, Tsujimoto M, Sakaeda $\mathrm{T}$ and Yokoyama T: Factors affecting sensitivity to antitumor platinum derivatives of human colorectal tumor cell lines. Cancer Chemother Pharmacol 62(4): 577-584, 2008. PMID: 18030470. DOI: $10.1007 / \mathrm{s} 00280-007-0640-3$

28 Klanova M, Andera L, Brazina J, Svadlenka J, Benesova S, Soukup J, Prukova D, Vejmelkova D, Jaksa R, Helman K, Vockova P, Lateckova L, Molinsky J, Maswabi BC, Alam M, Kodet R, Pytlik R, Trneny M and Klener P: Targeting of BCL2 family proteins with ABT-199 and homoharringtonine reveals BCL2- and MCL1-dependent subgroups of diffuse large B-cell lymphoma. Clin Cancer Res 22(5): 1138-1149, 2016. PMID: 26467384. DOI: 10.1158/1078-0432.CCR-15-1191

29 Deng W, Clipson A, Liu H, Huang Y, Dobson R, Wang M, Johnson $\mathrm{P}$ and Du MQ: Variable responses of MYC translocation positive lymphoma cell lines to different combinations of novel agents: Impact of BCL2 family protein expression. Transl Oncol 11(5): 1147-1154, 2018. PMID: 30055346. DOI: 10.1016/j.tranon.2018. 07.007

30 Pham LV, Huang S, Zhang H, Zhang J, Bell T, Zhou S, Pogue E, Ding Z, Lam L, Westin J, Davis RE, Young KH, Medeiros LJ, Ford RJ, Nomie K, Zhang L and Wang M: Strategic therapeutic targeting to overcome venetoclax resistance in aggressive B-cell lymphomas. Clin Cancer Res 24(16): 3967-3980, 2018. PMID: 29666304. DOI: 10.1158/1078-0432.CCR-17-3004

31 Esteve-Arenys A and Roue G: BFL-1 expression determines the efficacy of venetoclax in MYC+/BCL2+ double hit lymphoma. Oncoscience 5(3-4): 59-61, 2018. PMID: 29854869. DOI: 10.18632/oncoscience.402
32 Esteve-Arenys A, Valero JG, Chamorro-Jorganes A, Gonzalez D, Rodriguez V, Dlouhy I, Salaverria I, Campo E, Colomer D, Martinez A, Rymkiewicz G, Pérez-Galán P, Lopez-Guillermo A and Roué G: The BET bromodomain inhibitor CPI203 overcomes resistance to ABT-199 (venetoclax) by downregulation of BFL1/A1 in in vitro and in vivo models of MYC+/BCL2+ double hit lymphoma. Oncogene 37(14): 1830-1844, 2018. PMID: 29353886. DOI: $10.1038 / \mathrm{s} 41388-017-0111-1$

33 Haselager MV, Kielbassa K, Ter Burg J, Bax DJC, Fernandes SM, Borst J, Tam C, Forconi F, Chiodin G, Brown JR, Dubois J, Kater $\mathrm{AP}$ and Eldering E: Changes in Bcl-2 members after ibrutinib or venetoclax uncover functional hierarchy in determining resistance to venetoclax in CLL. Blood 136(25): 2918-2926, 2020. PMID: 32603412. DOI: 10.1182/blood.2019004326

34 Weiss J, Gajek T, Köhler BC and Haefeli WE: Venetoclax (ABT199) might act as a perpetrator in pharmacokinetic drug-drug interactions. Pharmaceutics 8(1): 5, 2016. PMID: 26927160. DOI: $10.3390 /$ pharmaceutics8010005

35 Wang JQ, Li JY, Teng QX, Lei ZN, Ji N, Cui Q, Zeng L, Pan Y, Yang DH and Chen ZS: Venetoclax, a BCL-2 inhibitor, enhances the efficacy of chemotherapeutic agents in wild-type ABCG2overexpression-mediated MDR cancer cells. Cancers (Basel) 12(2): 466, 2020. PMID: 32085398. DOI: 10.3390/cancers 12020466

Received July 7, 2021

Revised July 24, 2021

Accepted July 28, 2021 